\title{
Lateral indium-indium pair correlations within the wetting layers of buried InAs/GaAs quantum dots
}

\author{
B. Shin, B. Lita, and R. S. Goldman ${ }^{\text {a) }}$ \\ Department of Materials Science and Engineering, University of Michigan, Ann Arbor, \\ Michigan 48109-2136 \\ J. D. Phillips and P. K. Bhattacharya \\ Department of Electrical Engineering and Computer Science, University of Michigan, Ann Arbor, \\ Michigan 48109-2122
}

(Received 2 April 2002; accepted for publication 26 June 2002)

\begin{abstract}
We have investigated lateral In-In pair correlations within the wetting layers of buried InAs/GaAs quantum dots imaged with cross-sectional scanning tunneling microscopy. We quantified the number of In-In pairs along the [110] direction as a function of the spacing between them. Since the number of In-In pairs exceeds that of a randomly generated distribution of In atoms, significant lateral In clustering within the wetting layers is apparent. A comparison of the experimentally determined and randomly generated In-In pair distributions reveals nearest-neighbor In-In pair interaction energies similar to those calculated for InGaAs alloy surfaces [J. -H. Cho, S. B. Zhang, and A. Zunger, Phys. Rev. Lett. 84, 3654 (2000)]. The In-In pair correlations increase with high temperature annealing, indicating that vertical In-Ga interdiffusion occurs simultaneously with lateral In segregation. Together, these results suggest that initial In clustering in the wetting layer may be frozen at the surface during growth, and that annealing increases the effective sizes of these clusters, driving the system toward its segregated equilibrium state. (C) 2002 American Institute of Physics. [DOI: 10.1063/1.1501760]
\end{abstract}

Recently, arrays of stacked quantum dots have shown significant promise for a variety of novel device applications. ${ }^{1}$ In the InAs/GaAs system, the regions between the InAs islands, often termed the "wetting layers," contain a few layers of sparsely populated In atoms within a GaAs matrix. A detailed understanding of the interactions and dynamics of In atoms in the wetting layers is critical for optimally designed device applications of self-assembled InAs/ GaAs quantum dots. For example, the responsivity in quantum dot-based infrared detectors might be improved by eliminating the wetting layers while retaining the vertical and lateral separation of dots within a quantum dot superlattice structure. Furthermore, in the past few years, several theoretical and experimental efforts have elucidated nonrandomness phenomena such as clustering, ordering, and segregation in III-V compound semiconductor alloys. ${ }^{2-10} \mathrm{In}$ InGaAs alloys, these issues are beginning to be explored in non-dilute alloys far beyond the percolation limit, such as the wetting layers of buried InAs/GaAs quantum dots. For example, we recently reported direct measurements of vertical In-Ga interdiffusion and In surface segregation lengths within the wetting layers of buried InAs/GaAs quantum dots. ${ }^{11,12}$ Here, we report on lateral In-In pair correlations in InGaAs alloys beyond the percolation limit, using an analysis of cross-sectional scanning tunneling microscopy (XSTM) images of the wetting layers of buried InAs/GaAs quantum dots, in comparison with randomly generated In-In pair distributions. Our results reveal significant lateral In clustering in the wetting layers, which is likely initially frozen at the surface during growth. The extent of indium clus-

a)Electronic mail: rsgold@engin.umich.edu tering increases with annealing, as the system is driven towards its equilibrium, segregated state. Similar phenomena are expected to occur in a wide range of alloy systems.

The samples were grown by solid-source molecularbeam epitaxy, as described in an earlier report. ${ }^{13}$ The InAs/ GaAs quantum dots consisted of 2.6 monolayers of InAs, followed by $5 \mathrm{~nm} \mathrm{GaAs}$, with a $10 \mathrm{~s}$ interrupt under $\mathrm{As}_{4}$ flux separating their growth at $510^{\circ} \mathrm{C}$. The samples were effectively in situ annealed at $620^{\circ} \mathrm{C}$ for $22.5 \mathrm{~min}$. Ex situ postgrowth rapid-thermal-annealing was performed in argon gas at $900{ }^{\circ} \mathrm{C}$ for $30 \mathrm{~s}$. We will refer to the in situ and ex situ annealed wetting layers as "low $T$ " and "high $T$ " annealed wetting layers, respectively.

The spatial distribution of In atoms was obtained from an analysis of several high-resolution XSTM images, spanning areas greater than $2000 \mathrm{~nm}^{2}$, for both the low $T$ annealed and high $T$ annealed wetting layers, respectively. ${ }^{12}$ Figure 1 shows an example image, acquired at a positive sample bias voltage (empty states), where the cation sublattice is resolved, and the bright spots correspond to In atoms in a GaAs matrix. ${ }^{7,8,11}$ In each image, we used an algorithm to identify In and Ga atoms in bilayers within $12 \mathrm{~nm}$ of the atomic row with maximum In concentration. ${ }^{11,12,14}$ We estimated an In atom tip height criterion of $0.85 \AA \pm 0.05 \AA$ with respect to the GaAs background. ${ }^{14}$ For both the low $T$ and high $T$ annealed wetting layers, the average In concentration was $24 \pm 2 \%$. Using the experimentally determined In and $\mathrm{Ga}$ atom positions, we counted the number of In-In pairs, $N_{\text {In-In }}$ along the [110] direction, as a function of pair spacing, producing the open and filled circles in the plots in Figs. 2(a) and 2(b), for the low $T$ annealed and high $T$ annealed wetting layers, respectively. For comparison, random In-In pair 

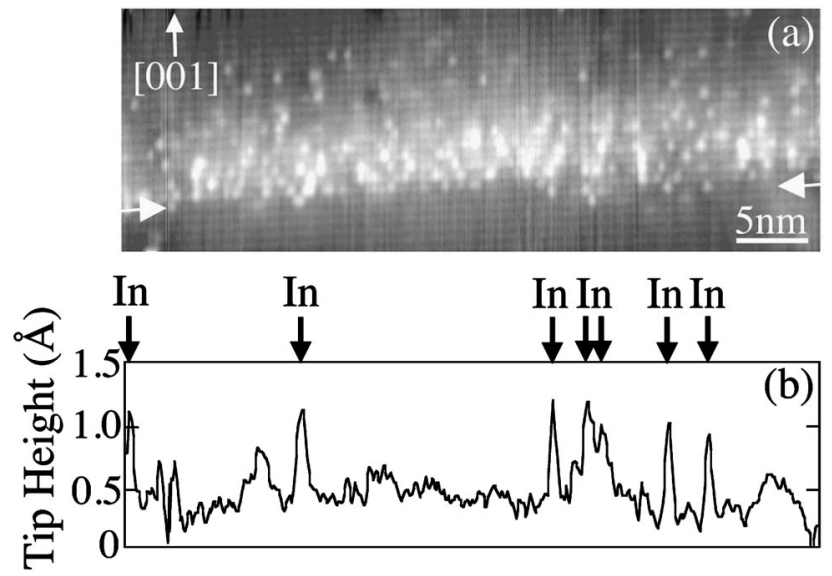

FIG. 1. (a) High-resolution XSTM topographic image of the wetting layers acquired at a sample bias voltage of $+2.4 \mathrm{~V}$. The gray-scale range displayed is $2.8 \AA$. A cut along the line indicated by arrows in (a) is displayed in (b). Pixels with tip height of $0.85 \AA \pm 0.05 \AA$ above the GaAs background were considered to be In atoms and are marked by the downward pointing arrows in (b).

distributions were generated as follows. Within each bilayer, we determined the fraction of sites occupied by In atoms and distributed them randomly. Using these computer-generated random distributions of In atoms, we then counted the number of In-In pairs along the [110] direction, as a function of spacing between them. We generated more than 4000 ran-
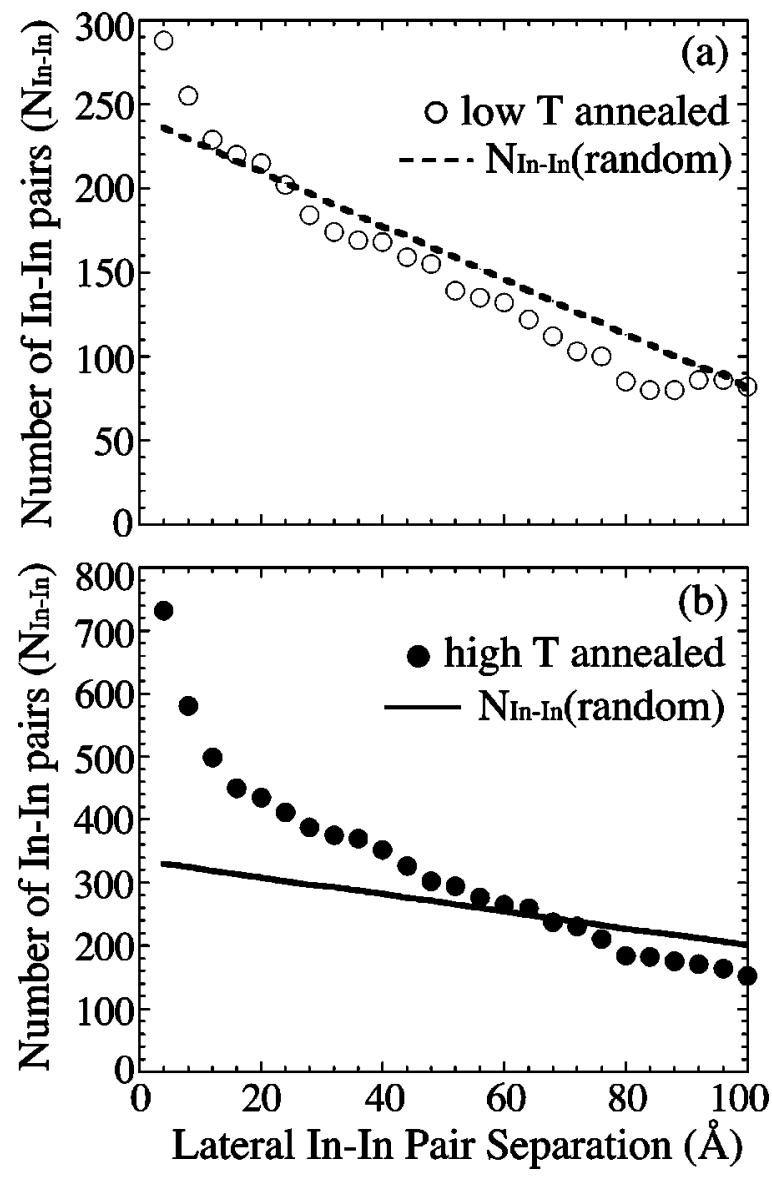

FIG. 2. Plot of number of In-In pairs vs In-In pair separation of (a) low $T$ annealed and (b) high $T$ annealed wetting layers. The dashed line in (a) and the solid line in (b) represent the number of In-In pairs for the computergenerated random distributions of In atoms for the low $T$ and high $T$ annealed wetting layers, respectively. dom distributions and averaged the number of In-In pairs to produce $N_{\text {In-In }}($ random), shown as the dashed and solid lines in Figs. 2(a) and 2(b), for the low $T$ and high $T$ annealed wetting layers, respectively.

For both the low $T$ and high $T$ annealed wetting layers shown in Figs. 2(a) and 2(b), $N_{\text {In-In }}$ and $N_{\text {In-In }}$ (random) decrease with increasing In-In pair separation. These decreases are due in part to the finite sizes of the images examined. In addition, for both the low $T$ and high $T$ annealed wetting layers, $N_{\text {In-In }}$ exhibits an oscillation with a period of $\sim 4-5$ lattice sites. This oscillation is likely related to the periodicity of the surface reconstruction during growth, similar to that suggested by Chao et al. ${ }^{9}$

For the low $T$ annealed wetting layers, $N_{\text {In-In }}$ is significantly larger than $N_{\text {In-In }}$ (random) for the first and second nearest-neighbors, suggesting the presence of short-range In-In pair correlations. However, beyond the second nearestneighbor, $N_{\text {In-In }}$ is either similar to or smaller than $N_{\text {In-In }}$ (random), suggesting the absence of long-range In-In pair correlations in the low $T$ annealed wetting layers.

In the case of the high $T$ annealed wetting layers, for In-In pair separations out to the 14th nearest-neighbor, $N_{\text {In-In }}$ is larger than $N_{\text {In-In }}$ (random), suggesting the presence of attractive In-In pair correlations over length-scales ranging from the first to the 14th nearest-neighbors. The pair separation beyond which $N_{\text {In-In }}$ (random) surpasses $N_{\text {In-In }}$ increases from six lattice sites for the low $T$ annealed wetting layers to 17 lattice sites for the high $T$ annealed wetting layers, indicating more significant lateral clustering after high $T$ annealing. In alloys, short-range deviations from randomness are often described by a clustering parameter, $\beta$, whereby the sign of $\beta$ indicates the tendency for clustering $(\beta>0)$ or anticlustering $(\beta<0) .{ }^{3}$ For InGaAs and related compound semiconductor alloys, $\beta$ is positive, indicating that clustering is the equilibrium state. ${ }^{15}$ Thus, our observation of increased In-In pair correlations with annealing suggests that the system is being driven towards its equilibrium clustered state.

The increase in In-In pair correlations with annealing also implies that vertical In-Ga interdiffusion occurs simultaneously with lateral In segregation. Fick's First Law describes a diffusional flux driven solely by a concentration gradient, without contributions from additional potential gradients. ${ }^{16}$ In our case, the diffusional flux needs to be modified to include a term due to lateral In-In segregation, which is dependent on the In-In pair interaction energy, $E_{\text {int }}$ :

$$
E_{\text {int }}=-k T \ln \left[N_{\text {In-In }} / N_{\text {In-In }}(\text { random })\right],
$$

where $k$ is the Boltzmann constant and $T$ is the absolute temperature. ${ }^{9}$ Using the $N_{\text {In-In }}$ and $N_{\text {In-In }}$ (random) from Figs. 2(a) and 2(b), we calculated $E_{\text {int }}$ as a function of In-In pair separation. We note that when $E_{\text {int }}=0$, the In-In pair interactions are negligible, and Fick's First Law is considered to describe the diffusional flux of the system. In Fig. 3, we plot $E_{\text {int }}$ as a function of In-In pair separation, and compare these data with previous experimental and theoretical studies of dilute InGaAs alloys. In the plot, positive and negative values of $E_{\text {int }}$ indicate repulsive and attractive interactions between neighboring In atoms. For both the low $T$ and high $T$ 


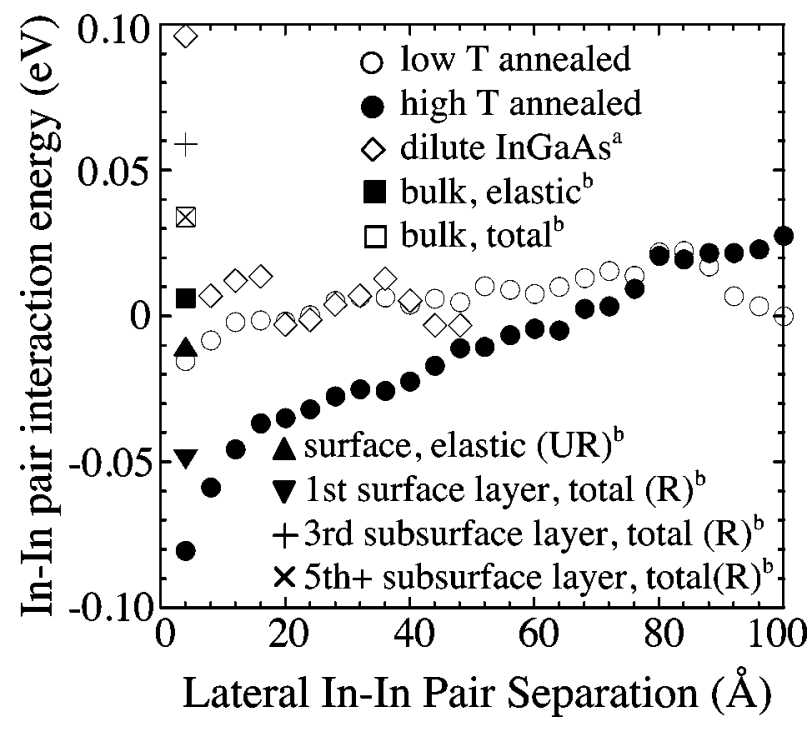

FIG. 3. Plot of In-In pair interaction energy vs In-In pair separation. Both experimentally and theoretically determined interaction energies of dilute InGaAs alloys are also shown. The calculated values denoted by "total" include both elastic and chemical effects; " $\mathrm{R}$ " and "UR" refer to reconstructed and unreconstructed surface structures, respectively. ${ }^{\text {a See Ref. } 9 .}$ ${ }^{\mathrm{b}}$ See Ref. 6.

annealed wetting layers, $E_{\text {int }}$ oscillates with a period of $\sim 4-5$ lattice sites. The oscillation is likely related to the periodicity of the surface reconstruction during growth discussed earlier.

In the case of low $T$ annealed wetting layers, $E_{\text {int }}$ is negative for the first few nearest-neighbors, but increases to zero for the third to sixth nearest-neighbors, and finally oscillates about a positive value for nearest-neighbors beyond the seventh. The significant decrease in attractive In-In pair interactions with In-In pair separation suggests the presence of strong short-range In segregation but weak long-range In-In repulsion. For comparison, an earlier experimental study of dilute InGaAs alloys, with In concentration $\sim 5 \%$, is also shown in the same plot. In that case, a strong repulsive first nearest-neighbor In-In pair interaction is evident. Beyond the first nearest-neighbor, a similar weak, oscillatory repulsive interaction occurs. Thus, in the dilute InGaAs alloy, there is a strong short-range In-In repulsion and a weak long-range In-In repulsion. On the other hand, in the high $T$ annealed wetting layers, $E_{\text {int }}$ is negative out to 16th nearestneighbor In pair, suggesting the presence of both short- and long-range In segregation.

For the low $T$ annealed and high $T$ annealed wetting layers, the nearest-neighbor In-In pair interaction energies, $E_{\text {int }}(\mathrm{NN})$, are $-0.015 \mathrm{eV}$ and $-0.081 \mathrm{eV}$, respectively. Interestingly, $E_{\text {int }}(\mathrm{NN})$ for the low $T$ annealed wetting layers lies within $5 \mathrm{meV}$ of that calculated for an unreconstructed InGaAs alloy surface. ${ }^{6}$ This similarity in values of measured and computed $E_{\text {int }}(\mathrm{NN})$ suggests that initial lateral In clustering in the InAs wetting layer may be frozen at the surface during growth. Furthermore, the more negative value of
$E_{\text {int }}(\mathrm{NN})$ for the high $T$ annealed wetting layers in comparison with the low $T$ annealed wetting layers suggests a stronger attractive interaction between the In atoms in that case. As mentioned earlier, the increase in attractive In-In interactions with annealing suggests that annealing drives the system toward its equilibrium state of clustering.

In summary, we investigated In-In pair correlations within the wetting layers of buried InAs/GaAs quantum dots imaged with XSTM. We quantified the number of In-In pairs as a function of the In-In pair spacing along the [110] direction. The experimentally determined number of In-In pairs exceeds that of a randomly generated distribution, suggesting the presence of significant lateral In clustering within the wetting layers. Interestingly, the nearest-neighbor In-In pair interaction energies are similar to those calculated for InGaAs alloy surfaces. Since the In-In pair correlations increase with high temperature annealing, lateral In segregation occurs simultaneously with In-Ga interdiffusion, as the system is driven toward its segregated equilibrium state.

This work was supported in part by the National Science Foundation (Grant No. 9773707), and the DoD Multidisciplinary University Research Initiative (MURI) administered by the Army Research Office under Grant No. DAAD 19-011-0462. The authors acknowledge D. J. Srolovitz and A. Zunger for useful discussions, and C. Huh and L. Mitcham for assistance with data analysis.

${ }^{1}$ See for example, D. Bimberg, M. Grundmann, and N. N. Ledentsov, Quantum Dot Heterostructures (Wiley, Chichester, 1999), pp. 277-302, and references therein.

${ }^{2}$ M. L. Seaford, W. Wu, K. G. Eyink, D. H. Tomich, J. R. Tucker, and L. F. Eastman, J. Vac. Sci. Technol. B 15, 1274 (1997).

${ }^{3}$ A. Zunger and S. Mahajan, in Handbook on Semiconductors (NorthHolland, Amsterdam, 1994), Vol. 3, pp. 1399-1514.

${ }^{4}$ T. Kawai, H. Yonezu, Y. Ogasawara, D. Saito, and K. Pak, J. Appl. Phys. 74, 1770 (1993)

${ }^{5}$ K. Yamaguchi, Y. Yasuda, A. Kovacs, and P. B. Barna, J. Appl. Phys. 89, 217 (2001).

${ }^{6}$ J.-H. Cho, S. B. Zhang, and A. Zunger, Phys. Rev. Lett. 84, 3654 (2000).

${ }^{7}$ J. F. Zhang, J. D. Walker, M. B. Salmeron, and E. R. Weber, Phys. Rev. Lett. 72, 2414 (1994).

${ }^{8}$ M. Pfister, M. B. Johnson, S. F. Alvarado, H. W. M. Salemink, U. Marti, D. Martin, F. Morier-Genoud, and F. K. Reinhart, Appl. Phys. Lett. 67, 1459 (1995).

${ }^{9}$ K.-J. Chao, C. K. Shih, D. W. Gotthold, and B. G. Streetman, Phys. Rev. Lett. 79, 4822 (1997).

${ }^{10}$ H. A. McKay, R. M. Feenstra, T. Schmidtling, and U. W. Pohl, Appl. Phys. Lett. 78, 82 (2001).

${ }^{11}$ B. Lita, R. S. Goldman, J. D. Phillips, and P. K. Bhattacharya, Appl. Phys. Lett. 75, 2797 (1999).

${ }^{12}$ B. Lita, R. S. Goldman, J. D. Phillips, and P. K. Bhattacharya, Surf. Rev. Lett. 7, 539 (2000).

${ }^{13}$ B. Lita, R. S. Goldman, J. D. Phillips, and P. K. Bhattacharya, Appl. Phys. Lett. 74, 2824 (1999).

${ }^{14}$ B. Lita, Ph.D. thesis, University of Michigan, 2002.

${ }^{15}$ S. Yamazaki, M. Kishi, and T. Katoda, Phys. Status Solidi B 113, 421 (1982).

${ }^{16}$ P. Shewmon, Diffusion in Solids, 2nd ed. (The Minerals, Metals, and Materials Society, Warrendale, PA, 1989), p. 10. 